\title{
Intoxicação experimental por Indigofera suffruticosa em caprinos e ovinos ${ }^{1}$
}

\author{
Anna P.M. de Figueiredo ${ }^{2}$, Rosane M.T. Medeiros ${ }^{2 *}$, Francelicia P.M. Dantas ${ }^{2}$, Amélia \\ L.D. Leite ${ }^{2}$, Rafael A. Fighera ${ }^{3}$ e Franklin Riet-Correa ${ }^{2}$
}

\begin{abstract}
Figueiredo A.P.M., Medeiros R.M.T., Dantas F.P.M., Leite A.L.D., Fighera R.A. \& Riet-Correa F. 2012. [Experimental poisoning by Indigofera suffruticosa in goats and sheep.] Intoxicação experimental por Indigofera suffruticosa em caprinos e ovinos. Pesquisa Veterinária Brasileira 32(2):126-130. Hospital Veterinário, Centro de Saúde e Tecnologia Rural, Universidade Federal de Campina Grande, Campus de Patos, 58700-970 Patos, PB, Brazil.E-mail: rmtmed@uol.com.br

Indigofera suffruticosa is a weed, which causes hemolytic anemia and hemoglobinuria in cattle and, experimentally, anemia without hemoglobinuria in guinea pigs. With the objective to determinate the toxicity of I. suffruticosa to sheep and goats aerial parts of the plant were administrated to six goats and four sheep at daily doses of 10, 20 and 40g of fresh plant per kg body weight, during two to 24 days. Blood samples were collected daily for the determination of packed cell volume, hemoglobin concentrations, and red blood cells count. Urine was also collected daily for urine examination and observation of color changes. Osmotic fragility and blood concentrations of hemoglobin and methemoglobin were determined in one goat and one sheep. Anemia due to extravascular hemolysis, without hemoglobinuria, was observed in the experimental sheep and goats. Heinz bodies were observed in brilliant cresyl blue stained blood smears. There was total or partial recovery of the anemia in spite of the continued plant administration. Eight to 12 hours after collection a bluish pigment was observed in the urine. It is suggested that aniline is the toxic compound of I. suffruticosa responsible for the hemolysis.
\end{abstract}

INDEX TERMS: Poisonous plants, anemia, hemolytic anemia, extravascular hemolysis, plant poisoning.

RESUMO.- Indigofera suffruticosa é uma planta invasora, que causa anemia hemolítica com hemoglobinúria em bovinos e, experimentalmente, anemia sem hemoglobinúria em cobaios. $\mathrm{O}$ objetivo deste trabalho foi determinar a toxicidade de I. suffruticosa para caprinos e ovinos. Partes aéreas da planta foram administradas a seis caprinos e quatro ovinos em doses diárias de 10, 20 e $40 \mathrm{~g}$ por $\mathrm{kg}$ de peso vivo, durante períodos de 2 a 24 dias. Foram avaliados os parâmetros hematológicos (hematócrito, níveis de hemoglobina e contagem de hemácias) e foi coletada urina para urinálise e observação de variações na colora-

\footnotetext{
${ }^{1}$ Recebido em 19 de setembro de 2011.

Aceito para publicação em 10 de outubro de 2011.

${ }^{2}$ Hospital Veterinário, Campus de Patos da Universidade Federal de Campina Grande, Patos, PB 58700-000, Brazil. *Autor para correspondência: rmtmed@uol.com.br

${ }^{3}$ Laboratório de Patologia Veterinária, Departamento de Patologia, Universidade Federal de Santa Maria, Camobi, Santa Maria, RS 9710 5-900, Brasil.
}

ção. Em um caprino e um ovino foram realizados os testes de fragilidade osmótica, determinação de hemoglobina e metemoglobina e pesquisa de corpúsculos de Heinz. Foi comprovado que em caprinos e ovinos, I. suffruticosa causa anemia hemolítica sem hemoglobinúria com formação de corpúsculos de Heinz. Os animais recuperaram-se da anemia, total ou parcialmente, mesmo com a continuidade da administração da planta. Oito a 12 horas após a coleta observa-se pigmento azulado na urina. Sugere-se que o pigmento seja anilina ou algum metabolito dessa substância e que a anilina seja o princípio ativo responsável pela hemólise causada por I. suffruticosa.

TERMOS DE INDEXAÇÃO: Anemia, anemia hemolítica, hemólise extravascular, intoxicação por planta, plantas tóxicas.

\section{INTRODUÇÃO}

Indigofera suffruticosa (anil, anileira) é uma planta invasora, arbustiva, com até dois metros de altura, da família Papilionoideae, encontrada em todo o Nordeste. Originária 
das Antilhas e América Central é pouco exigente, nascendo em qualquer tipo de solo (Alzugaray \& Alzugaray 1988, Lorenzi 2000). Durante a época da chuva I. suffruticosa brota rapidamente, permanecendo verde durante todo o período chuvoso; na época seca, permanece viva, porém visualizando-se apenas os galhos e algumas vagens secas. Em anos de boa pluviosidade aparece em quantidade suficiente para causar surtos de intoxicação (Riet-Correa et al. 2009), na forma de uma doença hemolítica intravascular caracterizada por hemoglobinúria e anemia em bovinos (Barbosa Neto et al. 2001, Salvador et al. 2010). Experimentalmente, causa anemia hemolítica sem hemoglobinúria em cobaios (Cavia porcellus) (Salvador et al. 2011).

No semiárido do Nordeste do Brasil produtores mencionam a ocorrência de casos de intoxicação em ovinos e caprinos que pastejam em áreas invadidas por I. suffruticosa. A suspeita tem como base a presença de urina vermelha. O objetivo deste trabalho foi determinar se I. suffruticosa causa anemia hemolítica e hemoglobinúria em caprinos e ovinos.

\section{MATERIAL E MÉTODOS}

O experimento foi realizado no Hospital Veterinário (HV) da Universidade Federal de Campina Grande (UFCG), Centro de Saúde e Tecnologia Rural (CSTR), Campus de Patos, Paraíba, utilizando seis caprinos da raça Moxotó e cruzas e quatro ovinos da raça Santa Inês e cruzas, machos, com $10-20 \mathrm{~kg}$, de seis meses a um ano de idade, que permaneceram alojados em baias de alvenaria. Os animais foram vermifugados e, antes do início do experimento, passaram por um período de adaptação de uma semana. Durante o experimento foram alimentados com uma mistura de capim verde e concentrado (ração comercial $囚$ e farelo de milho e de trigo) em quantidade equivalente a 1,5\% do peso vivo (pv) e água à vontade.

As doses das partes aéreas de Indigofera suffruticosa administradas e o período de administração para cada animal experimental são apresentados no Quadro 1 . A planta foi coletada, no Campus de Patos, CSTR/UFCG. A coleta foi realizada diariamente, sempre pela manhã, durante todo o experimento, no período de junho de 2009 a agosto de 2010. Quando na falta de chuva, a planta era irrigada para mantê-la verde. Foram coletadas as partes aéreas da planta e administradas por via oral.

A cada dia antes da administração da planta era realizada avaliação clínica, coleta de sangue da veia jugular com uso de tubo com vácuo contendo anticoagulante (ácido etilenodiaminotetra-

\section{Quadro 1. Animais experimentais, doses de Indigofera suffruticosa administradas e início da anemia (hematócrito $\geq 20 \%$ )}

\begin{tabular}{cccccc}
\hline $\begin{array}{c}\text { Animal } \\
\text { No }\end{array}$ & Espécie & $\begin{array}{c}\text { Peso } \\
(\mathrm{kg})\end{array}$ & $\begin{array}{c}\text { Dose diária } \\
\text { (g/kg) }\end{array}$ & $\begin{array}{c}\text { Dias de } \\
\text { administração }\end{array}$ & $\begin{array}{c}\text { Início da anemia } \\
\text { (dias) }\end{array}$ \\
\hline 1 & Caprino & 17,0 & 10 & 24 & 6 \\
2 & Caprino & 14,5 & 10 & 24 & 3 \\
3 & Caprino & 14,4 & 20 & 20 & 3 \\
4 & Caprino & 11,0 & 20 & 20 & 3 \\
5 & Caprino & 14,5 & 20 & 13 & 7 \\
6 & Caprino & 13,5 & 40 & 2 & 1 \\
7 & Ovino & 17,0 & 10 & 12 & 3 \\
8 & Ovino & 16,0 & 20 & 12 & 5 \\
9 & Ovino & 17,0 & 20 & 5 & $\mathrm{SA}^{\mathrm{a}}$ \\
10 & Ovino & 17,0 & 40 & 2 & $\mathrm{SA}^{\mathrm{a}}$ \\
\hline
\end{tabular}

${ }^{\text {a Sem anemia. }}$ cético [EDTA]), para avaliação dos parâmetros hematológicos (hematócrito, níveis de hemoglobina e contagem de hemácias), e coleta de urina para urinálise. 0 hemograma e a urinálise foram realizados utilizando-se as técnicas descritas por Kerr (2003). No Caprino 5 e no Ovino 9 foi realizado o teste de fragilidade osmótica (Vallada 1999) e foi determinada a presença de hemoglobina e metemoglobina no plasma sanguíneo (Nauom 1997). Para pesquisa de corpúsculos de Heinz foram realizados esfregaços sanguíneos com sangue previamente incubado com azul cresil brilhante (Nauom 1997).

A urina foi coletada com o uso de coletor de urina infantil adaptado para pequenos ruminantes e, posteriormente, colocada em um pote coletor universal e avaliada por meio de fitas reagentes ${ }^{4}$. A coloração da urina do animal era observada, quando ainda no coletor infantil e várias vezes ao dia, por no máximo 12 horas. Para isso, o pote coletor com urina permanecia no laboratório à temperatura ambiente.

No Caprino 5 e no Ovino 9 a coleta e análise de sangue foi realizada três vezes ao dia, durante os dois dias de administração da planta (antes de administrar a planta, ao meio dia e no final da tarde), e duas vezes ao dia (manhã e final da tarde), nos dois dias subsequentes à administração da planta.

Foram avaliados os parâmetros de peso do animal, comportamento (apático, normal ou agitado), grau de hidratação (Grau 1[0-5\%]; Grau 2[5\%-10\%]; e Grau 3[acima de 10\%]), apetite, coloração das mucosas (rosa, pálida ou porcelana), temperatura retal, coloração da urina (amarelo citrino, vermelha ou azul), frequências cardíaca e respiratória, motilidade ruminal, peristaltismo intestinal e aspecto das fezes (normal ou pastoso).

\section{RESULTADOS}

Tanto os ovinos quanto os caprinos apresentaram, no início do experimento, alteração em alguns dos parâmetros clínicos avaliados. Foram observados apatia, desidratação de Grau 1 e hiporexia (diminuição do consumo de volumoso) nos primeiros dois a cinco dias após o início da ingestão, principalmente nos animais que ingeriram doses diárias de $20 \mathrm{~g} / \mathrm{kg} / \mathrm{pv}$. A coloração das mucosas variou de acordo com os parâmetros hematológicos, ficando mais pálidas quando ocorria anemia. A temperatura retal se manteve dentro dos parâmetros normais (entre $38,5^{\circ} \mathrm{C}$ e $39,2^{\circ} \mathrm{C}$ ). Os animais apresentaram taquicardia (120 batimentos/ minuto) e taquipnéia (40 respirações/minuto) apenas nos três primeiros dias do experimento; posteriormente as frequências cardíaca e respiratória se mantiveram dentro dos parâmetros normais. Todos os animais tiveram uma perda de peso de 0,5 a $1 \mathrm{~kg}$, entre o $50^{\circ}$ e $100^{\circ}$ dias após o início da administração e, posteriormente, os que permaneceram por um período maior no experimento, se recuperaram.

Os dados dos parâmetros hematológicos avaliados estão descritos nos Quadros 2 e 3. A partir do primeiro dia de administração da planta já se observou variação nesses parâmetros; no entanto, o início da anemia (hematócrito de $21 \%$ ou menor) variou entre o $3^{\circ}$ e $7^{\circ}$ dia de ingestão. 0 maior grau de anemia observado foi no Caprino 3, com hematócrito de $11 \%$, no $13^{\circ}$ dia de ingestão da planta. Nos demais caprinos, o nível menor de hematócrito variou de 17 a $18 \%$, entre os dias 11 e 18 . A maioria dos animais apresentou recuperação parcial do hematócrito, mesmo conti-

\footnotetext{
${ }^{4}$ Uriquest ${ }^{\circledR}$ Plus, Labtest.
} 
Quadro 2. Parâmetros hematológicos avaliados dos caprinos e ovinos que ingeriram Indigofera suffruticosa em doses diárias de 10 e $20 \mathrm{~g} / \mathrm{kg}$

\begin{tabular}{|c|c|c|c|c|c|c|c|c|c|c|c|c|c|c|c|c|c|c|c|c|c|c|c|c|c|}
\hline \multirow{2}{*}{$\begin{array}{l}\text { Animal } \\
\text { Dose }\end{array}$} & \multirow{2}{*}{$\begin{array}{l}\text { Parâmetros } \\
\text { avaliados }\end{array}$} & \multicolumn{24}{|c|}{ Dias nos que foi administrada a planta } \\
\hline & & 1 & 2 & 3 & 4 & 5 & 6 & 7 & 8 & 9 & 10 & 11 & 12 & 13 & 14 & 15 & 16 & 17 & 18 & 19 & 20 & 21 & 22 & 23 & 24 \\
\hline $\mathrm{C}^{\mathrm{a}} 1$ & Hematócrito (\%) & 27 & 26 & 24 & 22 & 23 & 21 & 19 & 18 & 18 & 19 & 19 & 19 & 19 & 18 & 18 & 17 & 17 & 18 & 21 & 22 & 22 & 22 & 22 & 24 \\
\hline \multirow[t]{2}{*}{$10 \mathrm{~g} / \mathrm{kg}$} & Hemoglobina (g/dl) & 8,3 & 9,0 & 9,6 & 8,0 & 8,3 & 7,5 & 7,3 & 8,0 & 8,8 & 7,0 & 7,6 & 7,4 & 7,2 & 7,1 & 6,7 & 6,3 & 6,6 & 5,6 & 8,2 & 9,1 & 8,8 & 9,3 & 9,2 & 8,9 \\
\hline & Eritrócitos $\left(\mathrm{x} 10^{6}\right)$ & 12,0 & 12,0 & 15,9 & 14,3 & 11,4 & 10,7 & 10,5 & 9,4 & 10,5 & 9,2 & 8,7 & 10,2 & 9,6 & 9,3 & 4,9 & 9,4 & 7,3 & 6,4 & 7,9 & 10,3 & 9,5 & 9,1 & 10,8 & 10,7 \\
\hline $\mathrm{C} 2$ & Hematócrito (\%) & 23 & 23 & 21 & 22 & 25 & 22 & 22 & 21 & 21 & 23 & 18 & 20 & 20 & 20 & 20 & 19 & 20 & 19 & 18 & 20 & 18 & 18 & 18 & 19 \\
\hline \multirow[t]{2}{*}{$10 \mathrm{~g} / \mathrm{kg}$} & Hemoglobina (g/dl) & 9,8 & 10,5 & 8,5 & 8,5 & 10,5 & 6,5 & 7,4 & 8,7 & 8,8 & 9,4 & 7,5 & 8,0 & 8,1 & 8,6 & 7,9 & 7,8 & 8,6 & 8,5 & 7,2 & 8,9 & 7,3 & 7,6 & 7,6 & 7,2 \\
\hline & Eritrócitos $\left(\mathrm{x} 10^{6}\right)$ & 11,4 & 13,2 & 13,0 & 14,3 & 16,7 & 15,7 & 16,8 & 12,5 & 14,1 & 13,9 & 14,9 & 14,9 & 14,4 & 11,6 & 12,7 & 12,3 & 11,5 & 12,7 & 14,1 & 14,0 & 10,4 & 13,7 & 13,5 & 13,5 \\
\hline C 3 & Hematócrito (\%) & 25 & 23 & 21 & 20 & 19 & 16 & 16 & 15 & 15 & 13 & 13 & 12 & 11 & 12 & 12 & 13 & 15 & 18 & 16 & 16 & & & & \\
\hline \multirow[t]{2}{*}{$20 \mathrm{~g} / \mathrm{kg}$} & Hemoglobina (g/dl) & 10,8 & 7,4 & 8,5 & 7,2 & 6,9 & 5,9 & 5,9 & 5,8 & 5,8 & 5,0 & 5,3 & 4,4 & 4,3 & 4,2 & 4,4 & 4,1 & 5,4 & 6,4 & 6,2 & 5,8 & & & & \\
\hline & Eritrócitos $\left(\mathrm{x} 10^{6}\right)$ & 13,8 & 11,8 & 13,7 & 9,1 & 9,8 & 9,1 & 8,7 & 7,9 & 7,5 & 6,9 & 7,2 & 6,6 & 4,0 & 7,0 & 6,0 & 7,0 & 5,9 & 8,8 & 8,1 & 8,1 & & & & \\
\hline C 4 & Hematócrito (\%) & 23 & 23 & 21 & 22 & 25 & 22 & 22 & 21 & 21 & 23 & 18 & 20 & 20 & 20 & 20 & 19 & 20 & 19 & 18 & 20 & & & & \\
\hline \multirow[t]{2}{*}{$20 \mathrm{~g} / \mathrm{kg}$} & Hemoglobina (g/dl) & 9,8 & 10,5 & 8,5 & 8,5 & 10,5 & 6,5 & 7,4 & 8,7 & 8,8 & 9,4 & 7,5 & 8,0 & 8,1 & 8,6 & 7,9 & 7,8 & 8,6 & 8,5 & 7,2 & 8,9 & & & & \\
\hline & Eritrócitos $\left(\mathrm{x} 10^{6}\right)$ & 11,4 & 13,2 & 13,0 & 14,3 & 16,7 & 15,7 & 16,8 & 12,5 & 14,1 & 13,9 & 14,9 & 14,9 & 14,4 & 11,6 & 12,7 & 12,3 & 11,5 & 12,7 & 14,1 & 14,0 & & & & \\
\hline $\mathrm{C} 5$ & Hematócrito (\%) & 23 & 26 & 22 & 24 & 23 & 25 & 21 & 24 & 21 & 21 & 22 & 23 & 20 & & & & & & & & & & & \\
\hline \multirow[t]{4}{*}{$20 \mathrm{~g} / \mathrm{kg}$} & Hemoglobina (g/dl) & 8,4 & 9,5 & 7,3 & 8,4 & 8,3 & 8,2 & 7,7 & 8,8 & 7,3 & 7,2 & 7,6 & 7,3 & 7,5 & & & & & & & & & & & \\
\hline & Eritrócitos (x 106) & 16,5 & 18,2 & 14,5 & 16,4 & 14,8 & 15,8 & 12,4 & 15,6 & 15,2 & 15,1 & 14,0 & 13,9 & 14,7 & & & & & & & & & & & \\
\hline & Metemoglobina (\%) & 6,4 & 3,7 & 7,3 & 6,8 & 3,8 & 4,0 & 5,1 & 3,9 & 7,7 & 6,9 & 6,0 & 13,5 & 6,3 & & & & & & & & & & & \\
\hline & F. Osmótica (\%)* & 77,7 & 83,3 & 77,7 & 77,7 & 77,7 & 77,7 & 83,3 & 77,7 & 77,7 & 72,2 & 77,7 & 77,7 & 77,7 & & & & & & & & & & & \\
\hline 07 & Hematócrito (\%) & 29 & 26 & 21 & 25 & 26 & 26 & 24 & 21 & 22 & 20 & 25 & 25 & & & & & & & & & & & & \\
\hline \multirow[t]{2}{*}{$10 \mathrm{~g} / \mathrm{kg}$} & Hemoglobina (g/dl) & 8,9 & 8,5 & 8,3 & 8,7 & 9,3 & 8,5 & 8,1 & 5,8 & 8,9 & 6,9 & 8,6 & 8,5 & & & & & & & & & & & & \\
\hline & Eritrócitos $\left(\mathrm{x} 10^{6}\right)$ & 8,5 & 8,4 & 8,3 & 7,5 & 8,3 & 8,0 & 7,0 & 6,3 & 7,0 & 5,8 & 7,2 & 8,4 & & & & & & & & & & & & \\
\hline 08 & Hematócrito (\%) & 30 & 28 & 25 & 23 & 21 & 21 & 21 & 25 & 21 & 25 & 24 & 23 & & & & & & & & & & & & \\
\hline \multirow[t]{2}{*}{$20 \mathrm{~g} / \mathrm{kg}$} & Hemoglobina $(\mathrm{g} / \mathrm{dl})$ & 10,5 & 9,8 & 8,8 & 7,7 & 7,2 & 7,0 & 7,5 & 8,4 & 7,2 & 8,6 & 7,5 & 7,6 & & & & & & & & & & & & \\
\hline & Eritrócitos $\left(\mathrm{x} 10^{6}\right)$ & 8,5 & 8,3 & 8,1 & 7,4 & 8,1 & 6,3 & 6,3 & 6,5 & 6,2 & 6,9 & 4,9 & 5,4 & & & & & & & & & & & & \\
\hline 09 & Hematócrito (\%) & 35 & 34 & 33 & 33 & 33 & & & & & & & & & & & & & & & & & & & \\
\hline \multirow[t]{4}{*}{$20 \mathrm{~g} / \mathrm{kg}$} & Hemoglobina (g/dl) & 10,1 & 12,0 & 12,4 & 11,5 & 11,8 & & & & & & & & & & & & & & & & & & & \\
\hline & Eritrócitos $\left(\mathrm{x} 10^{6}\right)$ & 11,9 & 10,2 & 11,0 & 10,7 & 11,7 & & & & & & & & & & & & & & & & & & & \\
\hline & Metemoglobina (\%) & 3,1 & 2,4 & 4,2 & 4,3 & 4,3 & & & & & & & & & & & & & & & & & & & \\
\hline & F. Osmótica (\%)* & 61,1 & 77,7 & 72,2 & 77,7 & 77,7 & & & & & & & & & & & & & & & & & & & \\
\hline
\end{tabular}

Quadro 3. Parâmetros hematológicos avaliados do caprino e ovino que ingeriram Indigofera suffruticosa na dose de 40g/kg/ pv durante dois dias

\begin{tabular}{|c|c|c|c|c|c|c|c|c|c|c|c|}
\hline \multirow[t]{2}{*}{ Animal } & \multirow[t]{2}{*}{ Parâmetros avaliados } & \multicolumn{10}{|c|}{ Dias de experimento } \\
\hline & & $\begin{array}{c}1 \\
\left(1^{\mathrm{a}} \text { coleta }\right)\end{array}$ & $\begin{array}{c}1 \\
\left(2^{\circ} \text { coleta }\right)\end{array}$ & $\begin{array}{c}1 \\
\left(3^{\mathrm{a}} \text { coleta }\right)\end{array}$ & $\begin{array}{c}2 \\
\left(1^{a} \text { coleta }\right)\end{array}$ & $\begin{array}{c}2 \\
\left(2^{\mathrm{a}} \text { coleta }\right)\end{array}$ & $\begin{array}{c}2 \\
\left(3^{a} \text { coleta }\right)\end{array}$ & $\begin{array}{c}3 \\
\left(1^{\mathrm{a}} \text { coleta }\right)\end{array}$ & $\begin{array}{c}3 \\
\left(2^{\mathrm{a}} \text { coleta }\right)\end{array}$ & $\begin{array}{c}4 \\
\left(1^{\mathrm{a}} \text { coleta) }\right.\end{array}$ & $\begin{array}{c}4 \\
\left(2^{\mathrm{a}} \text { coleta) }\right.\end{array}$ \\
\hline \multirow[t]{3}{*}{$C^{a}-6$} & Hematócrito (\%) & 21 & 18 & 19 & 19 & 18 & 19 & 20 & 20 & 18 & 20 \\
\hline & Hemoglobina (g/dl) & 7,5 & 6,7 & 7,2 & 7,6 & 7,1 & 7,3 & 11,0 & 10,0 & 8,1 & 9,2 \\
\hline & Eritrócitos $\left(\mathrm{x} 10^{6}\right)$ & 11,2 & 9,7 & 9,7 & 9,3 & 11,9 & 12,1 & 10,7 & 10,2 & 10,3 & 10,5 \\
\hline \multirow[t]{3}{*}{$0-10$} & Hematócrito (\%) & 36 & 35 & 36 & 36 & 35 & 36 & 36 & 36 & 36 & 36 \\
\hline & Hemoglobina (g/dl) & 12,3 & 12,1 & 12,2 & 11,8 & 12,4 & 12,6 & 12,3 & 12,4 & 12,1 & 12,3 \\
\hline & Eritrócitos $\left(\mathrm{x} 10^{6}\right)$ & 12,5 & 10,6 & 10,3 & 13,2 & 11,5 & 12,3 & 11,9 & 12,3 & 12,5 & 12,4 \\
\hline
\end{tabular}

${ }^{\mathrm{a}} \mathrm{C}=$ caprino, $\mathrm{O}=$ ovino.

nuando a ingerir a planta, porém até o final do experimento todos os animais apresentaram hematócrito abaixo da primeira avaliação. Em relação à hemoglobina, o menor valor observado foi de $4,1 \%$, no $16^{\circ}$ dia de ingestão da planta, no Caprino 3. 0 Ovino 7 apresentou o menor hematócrito (20\%) no dia 9. 0 Ovino 8 apresentou o menor hematócrito (21\%) do dia 5 até o dia 9. Posteriormente houve recuperação total nos dois ovinos. Os Ovinos 9 e 10 não apresentaram diminuição nos valores hematológicos.

0 teste de fragilidade osmótica demonstrou uma pequena oscilação de $77,7 \%$ para $83,3 \%$, no Caprino 5 e de $61,1 \%$ para $77,7 \%$ no Ovino 9 , no $1^{\text {o }}$ e $2^{\circ}$ - dia de ingestão de I. suffruticosa, respectivamente. Observou-se, também, aumento dos valores de metemoglobina, sendo o maior pico de 13,5\% no Caprino 5, no $12^{\circ}$ dia de administração da planta, e 4,3 \% no Ovino 9, no 5으 dia de administração. Foi observada a presença intensa de corpúsculos de Heinz nos esfregaços sanguíneos.

Em nenhum momento foi observada hemoglobinúria. A única anormalidade observada na urina foi uma pigmentação azulada que aparecia entre 8 e 12 horas após a coleta.
Nos Caprinos 1 e 2, o pigmento foi observado a partir do 15으 dia e nos Caprinos 3, 4 e 5 a partir do 6을. Nesses animais, a urina voltou ao normal dentro de 24 horas após o final da ingestão da planta. No Caprino 6, o pigmento foi visto já no $1^{0}$ dia e voltou ao normal no $2^{2}$ dia após cessada a ingestão da planta.

Nos Ovinos 7 e 8 a presença do pigmento foi observada no $3^{\circ}$ dia de ingestão de I. suffruticosa, desaparecendo no 9o dia, mesmo com a continuidade da ingestão da planta. No Ovino 10 não foi observada a presença do pigmento na urina.

\section{DISCUSSÃO}

Indigofera suffruticosa causou em ovinos e caprinos anemia, uma vez que se observou diminuição dos parâmetros hematológicos (hematócrito, hemoglobina e contagem de eritrócitos). Esta anemia é hemolítica, pois se observou a presença de metemoglobina e corpúsculos de Heinz nos eritrócitos. 0 fato de não haver hemoglobinúria indica que a hemólise é extravascular. Neste caso, os eritrócitos são destruídos pelo sistema fagocítico monocitário, principalmente no fígado e baço (Fighera 2001). 
A anemia causada por I. suffruticosa em ovinos foi reversível, pois, mesmo com a ingestão continuada da planta, os animais se recuperaram espontaneamente, com níveis de hematócrito acima de $21 \%$. No entanto, nos caprinos a recuperação da anemia foi parcial, uma vez que, com exceção do Caprino 1, todos os demais, ao final do experimento, apresentavam hematócrito menor do que em seu início $(\leq 20 \%)$.

A semelhança do que foi observado neste experimento, recuperação espontânea também ocorre em bovinos que ingerem I. suffruticosa (Barbosa Neto et al. 2001, Salvador et al. 2010) e Ditaxis desertorum (Tokarnia et al. 1997), e talvez se deva a modificações da flora ruminal que se adapta para alterar a estrutura química do princípio ativo da planta, ainda desconhecido. Recuperação parcial, seguida de ciclos de anemia e recuperação, é observada em bovinos intoxicados por plantas do gênero Brassica, que contém S-metilcisteína sulfóxido (SMCO), metabólito que no rúmen, por ação de bactérias, sofre metabolização em dimetil dissulfito, que causa hemólise (Cheeke 1998). A recuperação da anemia ocorre, provavelmente, devido a uma adaptação parcial dos microorganismos do rúmem produzindo menos dimetil dissulfito. Neste trabalho, os ovinos, que apresentaram leve anemia, tiveram recuperação mais rápida do que os caprinos, provavelmente por que são mais resistentes aos agentes que causam oxidação de eritrócitos quando comparados com os caprinos (Cheeke 1998, Rae 1999, Fighera 2001).

Na intoxicação por I. sufruticosa em cobaios foi observada anemia hemolítica, sem hemoglobinúria, porém os valores do hematócrito e da hemoglobina foram diminuindo continuamente, não se observando o comportamento de recuperação espontânea, visto nos ruminantes (Salvador et al. 2011). Nos monogástricos, o SMCO é absorvido no intestino, antes de chegar ao ceco, onde deveria ocorrer a fermentação bacteriana, desta forma, as plantas do gênero Brassica, causam anemia hemolítica em ruminantes, mas não em monogástricos (Cheeke 1998). A suscetibilidade de cobaios à intoxicação por I. sufruticosa sugere que o princípio ativo da planta não é o SMCO (Salvador et al. 2011).

Outro sinal clínico na intoxicação experimental por I. suffruticosa em caprinos e ovinos foi o pigmento azulado observado na urina, 8-12 horas após a coleta. Esse pigmento também foi observado na urina de cobaios, 8-10 horas após a urinação (Salvador et al. 2011), e em bovinos (Barbosa Neto et al. 2001, Salvador et al. 2010) e pode ser utilizado como parâmetro para diagnóstico diferencial da intoxicação por I. suffruticosa com outras causas de anemia hemolítica. No Brasil, há pelo menos outras três plantas que causam hemólise: Brachiaria radicans em bovinos (Gava et al. 2010); Allium cepa em búfalos (Borelli et al. 2009) e bovinos (Bernardino 2010); e Ditaxis desertorum, em bovinos (Tokarnia et al. 1997), mas em nenhuma delas foi relatada a presença de pigmento azulado na urina.

A presença de pigmento azulado na urina dos animais intoxicados por I. suffruticosa sugere que o principio ativo da planta seja a anilina ou algum derivado da mesma (Salvador et al. 2011). A anilina $\left(\mathrm{C}_{6} \mathrm{H}_{5} \mathrm{NH}_{2}\right)$, obtida inicialmente por processo de destilação destrutiva do Indigo ( $I$. suffruticosa) (Anônimo 2006), causa crise hemolítica tóxica (Williams \& Challis 1933, Hughes \& Treon 1954) e mete- moglobinemia em seres humanos (Smith \& Olson 1973, Anônimo 2001) e em cães (Pauluhn 2002).

Sabe-se que após a ingestão, a anilina é metabolizada no fígado (Smith \& Olson 1973, Kiese 1974, Khan et al. 1998) em fenilhidroxilamina (Harrison \& Jollow 1987) e nitrobenzeno (Von Jagow et al. 1966, Murayama 1960, Kiese 1966, Khan et al. 2000) e induz alterações no metabolismo dos eritrócitos, formação de corpúsculos de Heinz e redução de $50 \%$ nos níveis de eritrócitos circulantes, sequencialmente (Pauluhn 2004). 0 aumento na formação de corpúsculos de Heinz é resultante, pricipalmente, do maior número de grupamentos sulfidrila da hemoglobina (McLean et al. 1969). Esta alteração eritróide resulta da diminuição no metabolismo da síntese de nicotinamida adenina dinucleotídeo (NADH). O NADH é um nucleotídeo formado pela via da pentose fosfato (Murayama 1960) que atua como coenzima na redução do glutation pela glutation-redutase (Harvey 1989). Em casos de intoxicação por primaquina (Bolchoz et al. 2001) e fenacetina (Jensen \& Jollow 1991), os dois derivados conhecidos da anilina, a diminuição da NADH é acentuada, resultando na falha da atividade da glutation-redutase e, consequentemente, na incapacidade de redução do glutation. A ação indireta dessas drogas sobre a atividade da glutation-redutase faz com que a maior parte do glutation permaneça oxidado nos eritrócitos, levando à formação de corpúsculos de Heinz (Bowman et al. 2004). Após a formação das inclusões, os eritrócitos começam a serem retirados da circulação pelo sistema fagocítico mononuclear (hemólise extravascular) mediante exposição dos sítios antigênicos previamente protegidos nas criptas (Singh et al. 2007).

\section{CONCLUSÃO}

Pelos resultados deste trabalho conclui-se que Indigofera suffruticosa causa anemia hemolítica, sem hemoglobinúria, em caprinos e ovinos. No entanto, não deve ser descartada a possibilidade de que, ocasionalmente, a planta possa causar hemoglobinúria nestas espécies, já que há produtores que mencionam o fato de terem observado urina de cor vermelha em caprinos e, menos frequentemente, em ovinos pastejando em áreas invadidas pela planta.

Agradecimento-- Este trabalho foi financiado pelo Instituto Nacional de Ciência e tecnologia Para o Controle das intoxicação por Plantas, Processo CNPq 573534/2008-0

\section{REFERÊNCIAS}

Alzugaray D. \& Alzugaray K. 1988. Enciclopédia de plantas brasileiras. Editora Três, São Paulo, p.54.

Anônimo 2001. Aniline-Acute exposure guideline levels. Inhalation. Toxicology 13(Suppl.):7-42.

Anônimo 2006. Aniline. <http://www.britannica.com/EBchecked/topic/25473/aniline> Acessado 23 jul. 2011.

Barbosa Neto J.D., Oliveira C.M.C., Peixoto P.V., Barbosa I.B.P., Ávila S.C. \& Tokarnia C.H. 2001. Anemia hemolítica causada por Indigofera suffruticosa (Leg. Papilionoideae) em bovinos. Pesq. Vet. Bras. 21(1):18-22.

Bernardino J.N.N. 2010. Comunicação pessoal (Veterinário Autônomo, Tabira, Pernambuco).

Bolchoz L.J., Budinsky R.A., McMillan D.C. \& Jollow D.J. 2001. Primaquineinduced hemolytic anemia: formation and hemotoxicity of the arylhydroxylamine metabolite 6-methoxy-8-hydroxylaminoquinoline. J. Pharmacol. Exp. Ther. 297:509-515. 
Borelli B., Lucioli J., Furlan F.H., Hoepers P.G., Roveda J.F., Traverso S.D. \& Gava A. 2009. Fatal onion (Allium cepa) toxicosis in water buffalo (Bubalus bubalis). J. Vet. Diagn. Invest. 21(3):402-405.

Bowman Z.S., Oatis J.E.Jr , Whelan J.L., Jollow D.J. \& Mcmillan D.C. 2004. Primaquine-Induced Hemolytic Anemia: susceptibility of normal versus glutathione-depleted rat erythrocytes to 5-Hydroxyprimaquine. J. Pharmacol. Exp. Ther. 309(1):79-85.

Cheeke P.R. 1998. Natural Toxicants in Feeds, Forages, and Poisonous Plants. $2^{\text {nd }}$ ed. Danville, Illinois, p.302-306.

Fighera R.A. 2001. Anemia em Medicina Veterinária. Editora Pallotti, Santa Maria. 214p.

Gava A., Simone de Deus M.R., Branco J.V., Mondadori A.J. \& Barth A. 2010. Intoxicação espontânea e experimental por Brachiaria radicans (tanner-grass) em bovinos. Pesq. Vet. Bras. 30(3):255-259.

Harrison J.JR. \& Jollow D.J. 1987. Contribution of aniline metabolites to aniline induced methemoglobinemia. Mol. Pharmacol. 32:423-431.

Harvey J.W. 1989. Erythrocyte metabolism, p.185-273. In: Kaneko J.J. (Ed.), Clinical Biochemistry of Domestic Animals. ${ }^{\text {th }}$ ed. Academic Press, San Diego.

Hughes J.P. \& Treon J.F. 1954. Erythrocytic inclusion bodies in the blood of chemical workers. AMA Arch. Indust. Health 10:192.

Jensen C.B. \& Jollow D.J. 1991. The role of N-hydroxyphenetidine in phenacetininduced hemolytic anemia. Toxicol. Appl. Pharmacol. 111:1-12.

Khan M.F., Green S.M., Ansari G.A. \& Boor P.J. 1998. Phenylhydroxylamine: role in aniline-associated splenic oxidative stress and induction of subendocardial necrosis. Toxicol. Sci. 42(1):64-71.

Khan M.F., Wu X. \& Ansari G.A. 2000. Contribution of nitrosobenzene to splenic of aniline. J. Toxicol. Environ. Health A 60(4):263-73.

Kerr M.G. 2003. Exames Laboratoriais em Medicina Veterinária: Bioquímica clínica e hematologia. $2^{\underline{a}}$ ed. Rocca, São Paulo. 436p.

Kiese M. 1966. The biochemical production of ferrihemoglobin-forming derivates from aromatic amines, and mechanisms of ferrihemoglobin formation. Pharmacol. Rev. 18(3):1091-161.

Kiese M. 1974. Methemoglobinemia: A comprehensive treatise. CRC Press, Cleveland, OH. 260 p.

Lorenzi H. 2000. Plantas daninhas do Brasil: terrestres, aquáticas, parasitas, tóxicas e medicinais. $3^{\underline{a}}$ ed. Instituto Plantarum, Nova Odessa. 608p.
McLean S., Stamer G.A. \& Thomas J. 1969. Methaemoglobin formation by aromatic amines. J. Pharm. Pharmacol. 21(7):441-50.

Murayama M. 1960. The combining power of normal human hemoglobin for nitrosobenzene. J. Biolog. Chem. 235:1024-1028.

Nauom P.C. 1997. Hemoglobinopatias e talassemias. Sarvier, São Paulo, 171p.

Pauluhn J. 2002. Aniline-induced methemoglobinemia in dogs: pitfalls of route-to-route extrapolations. Inhalation Toxicology 14:959-973.

Pauluhn J. 2004. Subacute inhalation toxicity of aniline in rats: analysis of time-dependence and concentration-dependence of hematotoxic and splenic effects. Toxicol. Sci. 81:198-215.

Rae H.A. 1999. Onion toxicosis in a herd of beef cows. Can. Vet. J. 40: 55-57.

Riet-Correa F. Medeiros R.M.T., Pfister J., Schild A.L. \& Dantas A.F.M. 2009. Poisonings by plants, mycotoxins and related substances in Brazilian livestock. Palotti, Santa Maria. 246p.

Salvador I. S., Medeiros R.M.T., Pessoa C.R.M., Dantas A.F.M., Sucupira Júnior G. \& Riet-Correa F. 2010. Intoxicação por Indigofera suffruticosa (leg. papilionoideae) em bovinos. Pesq. Vet. Bras. 30(11):953-957.

Salvador I.S., Medeiros R.M.T., Pessoa C.R.M., Oliveira D.M., Duarte A.L.L., Fighera R.A. \& Riet-Correa F. 2011. Experimental poisoning of guinea pig (Cavia porcellus) with Indigofera suffruticosa. Toxicon 57:927-931.

Singh H., Purnell E. \& Smith C. 2007. Mechanistic study on aniline-induced erythrocyte toxicity. Arh Hig Rada Toksikol. 58:275-285.

Smith R.P. \& Olson M.V. 1973. Drug-induced methemoglobinemia. Semin. Hematol. 10:253-268.

Tokarnia C.H., Chagas B.R., Chagas A.D. \& Silva H.K. 1997. Anemia hemolítica causada por Ditaxis desertorum (Euphorbiaceae) em bovinos. Pesq. Vet. Bras. 17(3/4):112-116.

Valentovic M.A., Yahia T., Ball J.G., Hong S.K., Brown P.I. \& Rankin G.O. 1997. 3,4-Dicholoroaniline acute toxicity in male Fischer 344 rats. Toxicology 124:125-134.

Vallada E.P.1999. Manual de técnicas hematológicas. Atheneu, São Paulo. 423p.

Von Jagow R., Kies M. \& Renner G. 1966. Urinary excretion of N-hydroxy derivatives of some aromatic amines by rabbits, guinea pigs, and dogs. Biochem. Pharmacol. 15(12):1899-1910.

Williams J.R. \& Challis F.E. 1933. Methylene blue as an antidote for aniline dye poisoning: Case report with confirmatory experimental study. J. Clin. Med. 19:166 